\title{
Nonexistence of sparse triple systems over abelian groups and involutions
}

\author{
Yuichiro Fujiwara
}

Received: 18 February 2006 / Accepted: 26 February 2007 /

Published online: 17 April 2007

(C) Springer Science+Business Media, LLC 2007

\begin{abstract}
In 1973 Paul Erdôs conjectured that there is an integer $v_{0}(r)$ such that, for every $v>v_{0}(r)$ and $v \equiv 1,3(\bmod 6)$, there exists a Steiner triple system of order $v$, containing no $i$ blocks on $i+2$ points for every $1<i \leq r$. Such an STS is said to be $r$-sparse. In this paper we consider relations of automorphisms of an STS to its sparseness. We show that for every $r \geq 13$ there exists no point-transitive $r$-sparse STS over an abelian group. This bound and the classification of transitive groups give further nonexistence results on block-transitive, flag-transitive, 2-transitive, and 2-homogeneous STSs with high sparseness. We also give stronger bounds on the sparseness of STSs having some particular automorphisms with small groups. As a corollary of these results, it is shown that various well-known automorphisms, such as cyclic, 1-rotational over arbitrary groups, and involutions, prevent an STS from being high-sparse.
\end{abstract}

Keywords Steiner triple system $\cdot r$-Sparse $\cdot$ Automorphism

Mathematics Subject Classification (2000) 05B07 · 20B25

\section{Introduction}

A Steiner triple system $S$ of order $v$, briefly $\operatorname{STS}(v)$, is an ordered pair $(V, \mathcal{B})$, where $V$ is a finite set of $v$ elements called points, and $\mathcal{B}$ is a set of 3-element subsets of $V$ called blocks, such that each unordered pair of distinct elements of $V$ is contained in exactly one block of $\mathcal{B}$. Kirkman [25] proved that an $\operatorname{STS}(v)$ exists if and only if $v \equiv 1,3(\bmod 6) ;$ such orders are called admissible.

Y. Fujiwara ( $\varangle)$

Graduate School of Information Science, Nagoya University, Furo-cho, Chikusa-ku,

464-8601, Japan

e-mail: fujiwara@math.cm.is.nagoya-u.ac.jp 
Let $\mathrm{G}^{(3)}(n ; m)$ denote a 3-uniform hypergraph of $n$ vertices and $m$ edges, that is, 3-tuples. Since an $\operatorname{STS}(v)$ contains exactly $v(v-1) / 6$ triples, it can be considered a special $\mathrm{G}^{(3)}(v ; v(v-1) / 6)$. In 1973 Erdôs [12] conjectured that for $r \geq 4$ there is an integer $v_{0}(r)$ such that for every $v>v_{0}(r), v \equiv 1,3(\bmod 6)$, there exists a Steiner triple system on $v$ elements containing no $\mathrm{G}^{(3)}(k+2 ; k)$ for every $1<k \leq r$. Such an STS is said to be $r$-sparse. Since the same pair of points appear twice in every $\mathrm{G}^{(3)}(k+2 ; k)$ for $1<k \leq 3$, every $\operatorname{STS}(v)$ is 3-sparse. Obviously, every $r$-sparse $\operatorname{STS}(v), r>2$, is also $(r-1)$-sparse.

The Erdôs $r$-sparse conjecture and especially the problem of characterizing those $v$, for which there exists an $r$-sparse $\operatorname{STS}(v)$, have been studied for a long time. One direction is regarding the $r$-sparse conjecture as an extremal problem on hypergraphs. In fact, Erdôs posed the conjecture as a problem closely related to extremal set theory. Brown, Erdős and Sós [3] proved:

Theorem 1.1 (Brown, Erdős and Sós [3]) Let $\mathcal{L}(k, l)$ be the family of all nonisomorphic 3-uniform hypergraphs with $l$ edges on $k$ vertices, and let ex $(n, \mathcal{L}(k, l))$ be the largest positive integer $m$ such that there exists a triple system with $m$ triples on $n$ vertices containing no member of $\mathcal{L}(k, l)$. Then

$$
\operatorname{ex}(n, \mathcal{L}(k+2, k)) \leq \frac{1}{3} \cdot\left(n \cdot\left\lfloor\frac{k}{k+1} \cdot(n-1)\right\rfloor+1\right)
$$

Let $\mathcal{V}(k+2, k)=\bigcup_{j=2}^{k} \mathcal{L}(j+2, j)$. Using probabilistic methods, Lefmann, Phelps and Rödl [26] showed that for every positive integer $k, k \geq 2$, there exists a $c_{k}$ such that $\operatorname{ex}(n, \mathcal{V}(k+2, k)) \geq c_{k} \cdot n^{2}$. They also proved the following theorem:

Theorem 1.2 (Lefmann, Phelps and Rödl [26]) There exists a positive constant $c>$ 0 , such that every Steiner triple system of order $v$ contains $a \mathrm{G}^{(3)}(k+2 ; k)$ for some $k \leq c \cdot \frac{\log v}{\log \log v}$.

On the other hand, a lot of construction techniques for $r$-sparse STSs of particular small $r$ and similar combinatorial structures have been developed. $\mathrm{A}^{(3)}(k ; l)$, when appearing in a Steiner triple system, is often called a "configuration" in recent literature, and so we shall use the same term here.

A $(k, l)$-configuration in an STS is a set of $l$ blocks whose union contains precisely $k$ points. An STS is $r$-sparse if and only if it contains no $(k+2, k)$-configuration for every $1<k \leq r$. As well as in combinatorial design theory, 4- and 5-sparse STSs are also important in some applications to information theory (see, for example, Chee, Colbourn and Ling [6]; Johnson and Weller [22]; Vasic, Kurtas and Kuznetsov [34]; and Vasic and Milenkovic [33]), and hence constructions of an $r$-sparse STS are studied extensively from both sides.

Brouwer [2] constructed a 4-sparse $\operatorname{STS}(v)$ for all $v \equiv 3(\bmod 6)$. He refined the Erdős $r$-sparse conjecture for the case $r=4$ to assert that a 4-sparse STS $(v)$ exists for all $v \equiv 1$ or $3(\bmod 6)$ except $v=7$ and 13 . Many partial results had been developed for this conjecture (see Colbourn and Rosa [8]). In particular, by developing several new constructions, Ling, Colbourn, Grannell and Griggs [28] substantially extended the spectrum of 4-sparse Steiner triple systems; and, finally, Brouwer's conjecture was settled by Grannell, Griggs and Whitehead [19]: 
Theorem 1.3 (Grannell, Griggs and Whitehead [19]) There exists a 4-sparse STS(v) if and only if $v \equiv 1,3(\bmod 6)$ and $v \neq 7,13$.

Thus, Erdős' conjecture is true for $r=4$ and $v_{0}(4)=13$. Also Colbourn, Mendelsohn, Rosa and Širáň [9], Ling [27], the author [14, 15], and Wolfe [36] have developed several constructions for an STS avoiding (6,4)- and/or (7, 5)-configuration. In particular, Wolfe [36] proved that for every admissible order $v$, except for $v=9$, there exists an $\operatorname{STS}(v)$ containing no mitre configuration, which is one of the two $(7,5)$-configurations. Recently, Wolfe [35] constructed a 5-sparse STS for all $v \equiv 3$ (mod 6) and $v \geq 21$. He also proved that there exists a 5 -sparse STS for, in some sense, almost all admissible orders.

Let $S$ and $T$ be two subsets of $\boldsymbol{Z}^{+}=\{1,2,3, \ldots\}$. Define the arithmetic density of $S$ relative to $T$ as:

$$
d(S ; T)=\lim _{n \rightarrow \infty} \frac{|\{x \in S \cap T: x \leq n\}|}{|\{x \in T: x \leq n\}|} .
$$

Theorem 1.4 (Wolfe [35]) The arithmetic density of the spectrum of 5-sparse Steiner triple systems relative to the set of all admissible orders is 1.

However, little is known about the existence of an STS with higher sparseness. In fact, no example of $r$-sparse systems is realized for $r \geq 7$ (and $v>3$ ), and no affirmative answer to the $r$-sparse conjecture is known in this range.

Our primary focus in the current paper is on relations between group actions on an STS and its sparseness. Frequently, actions of a finite group on a Steiner triple system have helped us discover an $r$-sparse STS and develop a construction method. An automorphism of an $\operatorname{STS}(v)=(V, \mathcal{B})$ is a permutation on $V$ that maps each block in $\mathcal{B}$ to a block of $\mathcal{B}$, and the full automorphism group is the group of all automorphisms of the STS. A flag of an STS $(V, \mathcal{B})$ is a pair $(x, B)$ with $x \in V, B \in \mathcal{B}$ and $x \in B$.

An STS is said to be point-transitive if its full automorphism group contains a subgroup which acts transitively on the point set. Similarly, we say that an STS is block-transitive, flag-transitive, 2-transitive, or 2-homogeneous if its full automorphism group contains a subgroup which acts transitively on the blocks, flags, ordered pairs of points, or unordered pairs of points, respectively.

Some classical constructions of STSs involving regular actions of $\operatorname{GF}(q)$ on the point set generate 4- and 5-sparse STSs (see Colbourn and Rosa [8]). The direct product construction for 5-sparse STSs, developed by Ling [27], employs an abelian group which acts regularly on the point set.

Theorem 1.5 (Ling [27]) If there exist a point-transitive 5-sparse $\operatorname{STS}(v)$ over an abelian group, $v \equiv 1(\bmod 6)$, and a 5-sparse $\mathrm{STS}(w)$, then there exists a 5-sparse $\operatorname{STS}(v w)$.

Forbes, Grannell and Griggs [13] discovered a construction method for blocktransitive STSs and found examples of 6-sparse STSs, which have the highest sparseness at the time of writing. They also developed a recursive construction similar to Theorem 1.5 for 6-sparse STSs and constructed infinitely many examples of such STSs. No 6-sparse STS other than from these Steiner triple systems is known. 
Also, when examining properties of an STS with computers, group actions often simplify calculations. In fact, by checking for $r$-sparseness the block-transitive STSs arising from one of known constructions, Forbes, Grannell and Griggs [13] found the first examples of 6-sparse STSs. By limiting the search to point-transitive STS $(v)$ over cyclic groups, that is, cyclic STS $(v)$, Colbourn, Mendelsohn, Rosa and Širán̆ [9] found a 5-sparse $\operatorname{STS}(v)$ for nearly all admissible $v<100$.

However, as we will see in the next section, such an STS can not have high sparseness. In this paper we prove first that, for every $r \geq 13$, there exists no pointtransitive $r$-sparse STS over an abelian group. This bound and the classification of transitive groups give further nonexistence results on block-transitive, flag-transitive, 2-transitive, and 2-homogeneous STSs with high sparseness. In Sect. 3 we give stronger bounds on sparseness of an STS admitting some particular automorphisms with small groups. As a corollary of these results, it is shown that various well-known automorphisms, such as cyclic, 1-rotational over arbitrary groups, and involutions with fixed points, prevent an STS from being highly-sparse. For undefined notions appearing in the following sections, we refer the reader to Beth, Jungnickel and Lenz [1], and Colbourn and Rosa [8].

\section{Transitive automorphism group}

In this section we consider transitive group actions on an STS and their relations to its sparseness. We examine transitive actions on points, blocks, flags, ordered pairs of points, and on an unordered pair of points. In what follows, we ignore the two trivial systems, that is, STS(1) and STS(3), unless they play a significant role.

First, we consider a point-transitive action. While the Erdős $r$-sparse conjecture says that, for any $r \geq 4$, an $r$-sparse STS $(v)$ exists for all sufficiently large admissible $v$, we show that every point-transitive STS over an abelian group is at most 12-sparse.

Theorem 2.1 For every $r \geq 13$, there exists no point-transitive $r$-sparse STS over an abelian group.

To verify Theorem 2.1 we prove two lemmas.

A point-transitive $\operatorname{STS}(V, \mathcal{B})$ over a group $G$ has a short orbit if there exist a block $B \in \mathcal{B}$ and an element $x \in G$ such that $B^{x}=B$ and $x \neq 1$, the identity element. $(V, \mathcal{B})$ has a $Z_{3}$-orbit if $\mathcal{B}$ contains a block of the form $\left\{a, a^{x}, a^{x^{2}}\right\}$, where $x^{3}=1$.

Lemma 2.2 Assume that there exists a point-transitive $r$-sparse STS over an abelian group $G$. If the STS has a $\mathbf{Z}_{3}$-orbit, then $r \leq 9$.

Proof Suppose to the contrary and let $S=(V, \mathcal{B})$ be a point-transitive $r$-sparse STS with $r>9$ over an abelian group $G$. In order to avoid the triviality, we assume that $|V|=v>3$. If $S$ has a $Z_{3}$-orbit, we can take a block $B_{1}=\left\{a, a^{x}, a^{x^{2}}\right\}$, where $a \in V$ and $x^{3}=1$. From $\mathcal{B} \backslash B_{1}$ take a block containing the point $a$ and write this block as $B_{2}=\left\{a, a^{i x}, a^{j x}\right\}$. Since $a^{i}, a^{j} \neq a, a^{x}, a^{x^{2}}$, we can find a block $B_{3}=\left\{a, a^{j}, a^{k}\right\}$ from the same family of blocks. By developing these three 
blocks, we obtain a possible $(12,10)$-configuration $B_{1}, B_{1}{ }^{i}, B_{1}{ }^{j}, B_{1}{ }^{k}, B_{2}, B_{2}{ }^{x}$, $B_{2}{ }^{x^{2}}, B_{3}, B_{3}{ }^{x}$ and $B_{3}{ }^{x^{2}}$. Since $i x, j \neq 1, x, x^{2}$, we have $B_{2}, B_{3} \notin \operatorname{Orb}_{G}\left(B_{1}\right)$. Hence, $\left|B_{1} \cup B_{1}{ }^{i} \cup B_{1}{ }^{j}\right|=\left|B_{1} \cup B_{1}{ }^{j} \cup B_{1}{ }^{k}\right|=9$. On the other hand, $r>9$ implies that $\left|B_{1} \cup B_{1}{ }^{i} \cup B_{1}{ }^{j} \cup B_{1}{ }^{k} \cup B_{2} \cup B_{2}{ }^{x} \cup B_{2}{ }^{x^{2}} \cup B_{3} \cup B_{3}{ }^{x} \cup B_{3}{ }^{x^{2}}\right|<12$. However, the only possible case is when $a^{k}=a^{x^{2} i} \Leftrightarrow a^{x k}=a^{i} \Leftrightarrow a^{x^{2} k}=a^{x i}$, leading to a (9,7)configuration $B_{1}, B_{1}{ }^{j}, B_{1}{ }^{k}, B_{2}, B_{3}, B_{3}{ }^{x}$, and $B_{3}{ }^{x^{2}}$. This contradicts the property that $r>9$. The proof is complete.

The following lemma concerns a point-transitive system having no $\boldsymbol{Z}_{3}$-orbit.

Lemma 2.3 Assume that there exists a point-transitive 6-sparse $\operatorname{STS}(v), S=(V, \mathcal{B})$, $v>3$, over an abelian group $G$. If $S$ has no $Z_{3}$-orbit, then $\mathcal{B}$ contains two blocks $B_{1}$ and $B_{2}$ such that these two blocks have the form $B_{1}=\left\{a, a^{x}, a^{x^{2} i}\right\}$ and $B_{2}=$ $\left\{a, a^{i}, a^{j}\right\}$, and $\operatorname{Orb}_{G}\left(B_{1}\right) \neq \operatorname{Orb}_{G}\left(B_{2}\right)$.

Proof Take an arbitrary block in $\mathcal{B}$ and write the triple as $\left\{a, a^{x}, a^{x^{2} i}\right\}$. Then we can find the block containing the pair of points $\left\{a, a^{i}\right\}$ and write this block as $\left\{a, a^{i}, a^{j}\right\}$. If the block orbits of these two blocks are different, our task is done. If the orbits are the same, then we have $i \in\left\{x, x i, x^{2} i, x^{-1},(x i)^{-1},\left(x^{2} i\right)^{-1}\right\}$. It is easy to see that the only possible cases are $i=x$ and $(x i)^{-1}$; otherwise, we have a pair of the same points in the block $\left\{a, a^{x}, a^{x^{2} i}\right\}$.

In the former case, rewrite the block $\left\{a, a^{x}, a^{x^{2} i}\right\}$ as $B_{1}=\left\{a, a^{x^{3}}, a^{x^{6} x^{-5}}\right\}$. If $a^{x^{-5}}=a$ then, by comparing $B_{1}$ and $B_{1}{ }^{x^{2}}$, we have $a^{x}=a^{x^{2}}$, that is, $a=$ $a^{x}$, a contradiction. Hence, we can find the block containing the pair $\left\{a, a^{x^{-5}}\right\}$ and write this block as $B_{2}=\left\{a, a^{x^{-5}}, a^{j}\right\}$. If $\operatorname{Orb}_{G}\left(B_{1}\right)=\operatorname{Orb}_{G}\left(B_{2}\right)$ then $x^{5} \in$ $\left\{x, x^{2}, x^{3}, x^{-1}, x^{-2}, x^{-3}\right\}$. In each case, we obtain the same pair of points twice in $\mathcal{B}$, or an $(r+2, r)$-configuration for some $r \leq 6$, a contradiction. Hence, we obtain a pair of blocks whose orbits are mutually different.

In the latter case, rewrite similarly the block $\left\{a, a^{x}, a^{x^{2} i}\right\}$ as $B_{1}=\left\{a, a^{i^{-3}}, a^{i^{-6} i^{4}}\right\}$. Following the above argument, we obtain the required pair of blocks again. This completes the proof.

We now return to the proof of Theorem 2.1.

Proof of Theorem 2.1 Suppose to the contrary that there exists a point-transitive $r$ sparse STS, $S=(V, \mathcal{B})$, over an abelian group $G$, where $r \geq 13$. By Lemma 2.2, $S$ has no $\boldsymbol{Z}_{3}$-orbit. Hence, by Lemma 2.3 , we can take a pair of blocks which have the form $B_{1}=\left\{a, a^{x}, a^{x^{2} i}\right\}$ and $B_{2}=\left\{a, a^{i}, a^{j}\right\}$, where $\operatorname{Orb}_{G}\left(B_{1}\right) \neq \operatorname{Orb}_{G}\left(B_{2}\right)$. Take a pair of blocks $B_{3}=\left\{a, a^{x j}, a^{x k}\right\}$ and $B_{4}=\left\{a, a^{k}, a^{l}\right\}$ in $\mathcal{B}$. By developing these four blocks, we obtain a possible $(15,13)$-configuration $B_{1}, B_{1}{ }^{i}, B_{1}{ }^{j}$, $B_{1}{ }^{k}, B_{1}{ }^{l}, B_{2}, B_{2}{ }^{x}, B_{2}{ }^{x^{2} i}, B_{3}, B_{3}{ }^{x i}, B_{4}, B_{4}{ }^{x}$, and $B_{4}{ }^{x^{2} i}$. Let $\alpha$ be the number of mutually different blocks in the possible $(15,13)$-configuration and $\beta$ be the number of mutually different points in the developed configuration, that is, $\beta=$ $\left|B_{1} \cup B_{1}{ }^{i} \cup B_{1}{ }^{j} \cup B_{1}{ }^{k} \cup B_{1}{ }^{l} \cup B_{2} \cup B_{2}{ }^{x} \cup B_{2}{ }^{x^{2} i} \cup B_{3} \cup B_{3}{ }^{x i} \cup B_{4} \cup B_{4}{ }^{x} \cup B_{4}{ }^{x^{2} i}\right|$. 
Since $r>12$, we must have $\alpha+2<\beta<15$ to avoid the developed configuration being an $(r+2, r)$-configuration.

First, we show that $\left|B_{1} \cup B_{1}{ }^{k} \cup B_{1}{ }^{l}\right|=9$. Suppose to the contrary that $\mid B_{1} \cup B_{1}{ }^{k} \cup$ $B_{1}^{l} \mid<9$. We consider six cases:

Case (1): $a=a^{x l}$. By considering the six blocks $B_{1}, B_{1}{ }^{k}, B_{1}{ }^{l}, B_{4}, B_{4}{ }^{x}$, and $B_{4}{ }^{x^{2} i}$, it is easy to see that the above condition is equivalent to $a^{k}=a^{x^{2} i l}$ and $a^{x k}=a^{x^{2} i}$. By noting the two blocks $B_{1}$ and $B_{3}$, we have $a^{x}=a^{x j}$, that is, $a=a^{j}$, a contradiction.

Case (2): $a=a^{x^{2} i l}$. As is Case (1), this equation is equivalent to $a^{k}=a^{x l} \Leftrightarrow$ $a^{x}=a^{x^{2} i k}$. By noting the two blocks $B_{2}{ }^{x}$ and $B_{3}{ }^{x i}$, we have $a^{x j}=a^{x^{2} i j}$. However, $\operatorname{Orb}_{G}\left(B_{1}\right) \neq \operatorname{Orb}_{G}\left(B_{2}\right)$ implies that $\left|B_{1} \cup B_{1}{ }^{i} \cup B_{1}{ }^{j}\right|=9$, a contradiction.

Case (3): $a=a^{x k}$. This is equivalent to $a^{l}=a^{x^{2} i k} \Leftrightarrow a^{x l}=a^{x^{2} i}$. Since $B_{3}=$ $\left\{a, a^{x j}, a^{x k}\right\} \in \mathcal{B}$, again a contradiction.

Case (4): $a=a^{x^{2} i k} \Leftrightarrow a^{l}=a^{x k} \Leftrightarrow a^{x}=a^{x^{2} i l}$. Take the seven blocks $B_{1}, B_{1}{ }^{i}$, $B_{1}{ }^{j}, B_{2}, B_{2}{ }^{x}, B_{2}{ }^{x^{2} i}$, and $B_{3}{ }^{x i}$. Since $\left|B_{1} \cup B_{1}{ }^{i} \cup B_{1}{ }^{j}\right|=9$, these seven blocks form a $(9,7)$-configuration, a contradiction.

Case (5): $a^{k}=a^{x} \Leftrightarrow a^{l}=a^{x^{2} i} \Leftrightarrow a^{x l}=a^{x^{2} i k}$. Consider the set of nine blocks $\mathcal{S}_{9}=\left\{B_{1}, B_{1}{ }^{i}, B_{1}{ }^{j}, B_{2}, B_{2}{ }^{x}, B_{2}{ }^{x^{2} i}, B_{3}, B_{3}{ }^{x i}, B_{4}{ }^{x}\right\}$. If $\left|\left\{B_{1} \cup B_{1}{ }^{i} \cup B_{1}{ }^{j}\right\} \cap B_{4}{ }^{x}\right|=1$, then $\mathcal{S}_{9}$ forms a $(11,9)$-configuration, a contradiction. Hence, $\mid\left\{B_{1} \cup B_{1}{ }^{i} \cup B_{1}{ }^{j}\right\} \cap$ $B_{4}{ }^{x} \mid \geq 2$. But then we obtain a $(9,7)$-configuration in $\mathcal{S}_{9} \backslash\left\{B_{3}, B_{4}{ }^{x}\right\}$, or in $\mathcal{S}_{9} \backslash$ $\left\{B_{3}{ }^{x i}, B_{4}{ }^{x}\right\}$, a contradiction.

Case (6): $a^{l}=a^{x} \Leftrightarrow a^{k}=a^{x^{2} i} \Leftrightarrow a^{x k}=a^{x^{2} i l}$. Consider the set of nine blocks $\mathcal{S}_{9}=\left\{B_{1}, B_{1}{ }^{i}, B_{1}{ }^{j}, B_{2}, B_{2}{ }^{x}, B_{2}{ }^{x^{2} i}, B_{3}, B_{3}{ }^{x i}, B_{4}{ }^{x}\right\}$. It reduces, by symmetry, to Case (5).

Next, we prove that $\left\{B_{1} \cup B_{1}{ }^{i} \cup B_{1}{ }^{j}\right\} \cap\left\{B_{1}{ }^{k} \cup B_{1}{ }^{l}\right\}=\emptyset$. Suppose to the contrary that $\left|\left\{B_{1} \cup B_{1}{ }^{i} \cup B_{1}{ }^{j}\right\} \cap\left\{B_{1}{ }^{k} \cup B_{1}{ }^{l}\right\}\right| \geq 1$. If $\left|\left\{B_{1} \cup B_{1}{ }^{i} \cup B_{1}{ }^{j}\right\} \cup\left\{a^{x k}, a^{x^{2} i k}\right\}\right|<11$, we obtain a $(9,7)$-configuration $B_{1}, B_{1}{ }^{i}, B_{1}{ }^{j}, B_{2}, B_{2}{ }^{x}, B_{2}{ }^{x^{2} i}$ and $B_{3}$ or $B_{3}{ }^{x i}$, a contradiction. Hence, we must have $\alpha \geq 12$. However, $\mid\left\{B_{1} \cup B_{1}{ }^{i} \cup B_{1}{ }^{j}\right\} \cap\left\{B_{1}{ }^{k} \cup\right.$ $\left.B_{1}^{l}\right\} \mid \geq 1$ implies that $\beta \leq 14$. This contradicts the inequality $\alpha+2<\beta<15$.

Thus, we have $\left|B_{1} \cup \bar{B}_{1}{ }^{k} \cup B_{1}{ }^{l}\right|=9$ and $\left\{B_{1} \cup B_{1}{ }^{i} \cup B_{1}{ }^{j}\right\} \cap\left\{B_{1}{ }^{k} \cup B_{1}{ }^{l}\right\}=\emptyset$. On the other hand, $\operatorname{Orb}_{G}\left(B_{1}\right) \neq \operatorname{Orb}_{G}\left(B_{2}\right)$ implies that $\left|B_{1} \cup B_{1}{ }^{i} \cup B_{1}{ }^{j}\right|=9$. Hence, we must have $\alpha=13$ and $\beta=15$, a contradiction. This completes the proof.

$\operatorname{An} \operatorname{STS}(v)$ is said to be cyclic if its automorphism group contains a cyclic group of order $v$ as a subgroup acting regularly on the point set. Peltesohn [29] proved that a cyclic $\operatorname{STS}(v)$ exists for all admissible $v$, except for $v=9$. Colbourn, Mendelsohn, Rosa and Širáň [9] found many examples of cyclic 5-sparse STSs using computers. The author [16] developed some general recursive constructions for cyclic 4- and 5sparse STSs, and constructed such an STS for infinitely many orders. However, by Theorem 2.1 and Lemma 2.2, we have:

Corollary 2.4 For every $r \geq 13$, there exists no cyclic $r$-sparse $\operatorname{STS}(v)$. In particular, when $v \equiv 3(\bmod 6)$, no cyclic $r$-sparse $\operatorname{STS}(v)$ exists for every $r \geq 10$.

Proof The first statement follows from Theorem 2.1. For a cyclic STS, the set of points can be identified with $\boldsymbol{Z}_{v}$, the residue group of integers modulo $v$. It is easy 
to see that every cyclic $\operatorname{STS}(v), v \equiv 3(\bmod 6)$, has the block $\{0, v / 3,2 v / 3\}$, which leads to a $\boldsymbol{Z}_{3}$-orbit.

In the rest of this section we briefly mention corollaries of the classification of STSs, admitting other types of transitive actions. By employing the classification of finite simple groups, Key and Shult [24], Hall [20] and Kantor [23] classified 2-transitive Steiner triple systems.

Theorem 2.5 (Key and Shult [24], Hall [20] and Kantor [23]) If a finite group G acts 2-transitively on the points of an STS $S$, then $S$ is either a projective space or an affine space.

Every projective space contains $(6,4)$-configurations, that is, they are not 4-sparse. On the other hand, every affine space is 4-sparse but not 5-sparse (see Colbourn and Rosa [8]). Hence, we obtain the following corollary:

Corollary 2.6 For every $r \geq 5$, there exists no 2-transitive $r$-sparse STS.

Delandtsheer, Doyen, Siemons and Tamburini [10] completed the classification for 2-homogeneous STSs.

Theorem 2.7 (Delandtsheer, Doyen, Siemons and Tamburini [10]) If a finite group $G$ acts 2-homogeneously but not 2-transitively on the points of an STS $S$, then $S$ is either an affine space over a subfield of $G F\left(p^{n}\right)$, where a prime $p \equiv 3(\bmod 4)$ and $n$ is odd, or a Netto triple system of order $p^{n} \equiv 7(\bmod 12)$.

Every Netto triple system of order $v, v \equiv 19(\bmod 24)$, is 5 -sparse, but none is 6-sparse (see Colbourn, Mendelsohn, Rosa and Širáň [9] and Robinson [30]). Hence, any 2-homogeneous STS is at most 5-sparse.

Corollary 2.8 For every $r \geq 6$, there exists no 2-homogeneous $r$-sparse STS.

The action of a finite group $G$ on an STS is flag-transitive if and only if it is 2-homogeneous on points (see Colbourn and Rosa [8]).

Corollary 2.9 For every $r \geq 6$, there exists no flag-transitive $r$-sparse STS.

For block-transitive STSs, Clapham [7] established the classification.

Theorem 2.10 (Clapham [7]) If a finite group $G$ acts block-transitively but not 2-transitively on the points of an STS $S$, then $G$ has odd order and is a subgroup of $A \Gamma L\left(1, p^{\alpha}\right)$ containing the translation group, for some prime $p$ and $\alpha \geq 1$, and one of the following holds:

1. $S$ is an affine space of dimension $d$ over $G F(3), d$ is odd, and $G$ has rank 3 on points;

2. $S$ is a Netto triple system; or

3. G has rank 7 on points and $p^{d} \equiv 7(\bmod 12)$. 
He also showed that if a finite group $G$ acts transitively on the block set of an STS, then it also acts transitively on the point set. Hence, by combining Theorem 2.1 and Corollary 2.6 with Theorem 2.10, we have the same bound.

\section{Corollary 2.11 For every $r \geq 13$, there exists no block-transitive $r$-sparse STS.}

Remark Any Steiner triple system of Case 1 and 2 in Theorem 2.10 is not 6-sparse. It is notable that the construction developed by Grannell, Griggs and Murphy [18] can generate finitely many examples of block-transitive 6-sparse STSs but none of them are 7-sparse (see Forbes, Grannell and Griggs [13]).

\section{Automorphism with fixed points}

In this section we mainly consider Steiner triple systems admitting a nontrivial automorphism with fixed points.

$\operatorname{An} \operatorname{STS}(v)$ is said to be 1-rotational over a group $G$ if it admits $G$ as a subgroup of the full automorphism group, and $G$ fixes exactly one point and acts regularly on the other points. A 1-rotational automorphism is closely related to an involution.

An STS is said to be reverse if it admits an involutory automorphism fixing exactly one point. Any 1-rotational STS is reverse. Indeed, for every 1-rotational STS $(v)$ over a group $G$, the order of $G$ is $v-1$ and even. Hence, $G$ has at least one involution.

Buratti [4] showed that there exists a 1-rotational STS $(v)$ over an abelian group if and only if $v \equiv 3,9(\bmod 24)$ or $v \equiv 1,19(\bmod 72)$. He also gave partial answers for an arbitrary group. The combined work of Doyen [11], Rosa [31] and Teirlinck [32] established the fact that the spectrum for reverse STS is the set of all $v \equiv 1,3,9$ or 19 (mod 24). An STS admitting an automorphism with more than one fixed point is known to exist (see Hartman and Hoffman [21]) and may also be considered. However, the fixed points must induce a smaller STS as a subsystem, and hence sparseness of the original Steiner system can not exceed that of the small sub-STS. Most interesting is the case when the induced subsystem is a trivial STS, that is, one point and no block, or three points and one block. The following theorem shows that such an STS is at most 4-sparse.

Theorem 3.1 For every $r \geq 5$, there exists no $r$-sparse STS admitting an involutory automorphism fixing exactly one or three points.

Proof Let $(V, \mathcal{B})$ be an STS. First, we consider an involution $\pi_{1}$ on $V$ fixing exactly one point, say $\infty$. Suppose to the contrary that $(V, \mathcal{B})$ is 5 -sparse and admits $\pi_{1}$ as an automorphism. If $\mathcal{B}$ contains a block which has the form $\{\infty, a, b\}$, where $b \neq \pi_{1}(a)$, then $\left\{\infty, \pi_{1}(a), \pi_{1}(b)\right\},\left\{a, \pi_{1}(b), c\right\},\left\{\pi_{1}(a), b, \pi_{1}(c)\right\} \in \mathcal{B}$, where $c \neq \pi_{1}(c)$. Since $(V, \mathcal{B})$ is an STS, a pair $\left\{c, \pi_{1}(c)\right\}$ must appear in exactly one block, say $\left\{c, \pi_{1}(c), d\right\}$. Then $\left\{c, \pi_{1}(c), \pi_{1}(d)\right\}$ is also in $\mathcal{B}$. This implies that $d=\pi_{1}(d)=\infty$. Hence, we obtain a $(7,5)$-configuration $\{\infty, a, b\},\left\{\infty, \pi_{1}(a), \pi_{1}(b)\right\},\left\{\infty, c, \pi_{1}(c)\right\},\left\{a, \pi_{1}(b), c\right\}$ and $\left\{\pi_{1}(a), b, \pi_{1}(c)\right\}$, a contradiction. If $\mathcal{B}$ contains no block of the form $\{\infty, a, b\}$, where $b \neq \pi_{1}(a), \mathcal{B}$ must have a pair of blocks $\{a, b, c\}$ and $\left\{\pi_{1}(a), \pi_{1}(b), \pi_{1}(c)\right\}$, where $c \neq \infty$. As noted above, each pair having the form $\left\{x, \pi_{1}(x)\right\}$ and the point 
$\infty$ appear together in $\mathcal{B}$. Hence, we obtain a $(7,5)$-configuration $\left\{\infty, a, \pi_{1}(a)\right\}$, $\left\{\infty, b, \pi_{1}(b)\right\},\left\{\infty, c, \pi_{1}(c)\right\},\{a, b, c\}$ and $\left\{\pi_{1}(a), \pi_{1}(b), \pi_{1}(c)\right\}$, a contradiction.

Next we consider an involution $\pi_{3}$ on $V$ fixing exactly three points. Suppose to the contrary that $(V, \mathcal{B})$ is 5-sparse and admits $\pi_{3}$ as an automorphism. As is the case with the involution $\pi_{1}$, a pair $\left\{a, \pi_{3}(a)\right\}$, where $a \neq \pi_{3}(a)$, must occur in a block with one of fixed points, say $\infty_{0}$. Considering the points $a, \pi_{3}(a)$ and another fixed point, say $\infty_{1}$, we have $\left\{\infty_{1}, a, b\right\},\left\{\infty_{1}, \pi_{3}(a) \pi_{3}(b)\right\} \in \mathcal{B}$. Again the pair $\left\{b, \pi_{3}(b)\right\}$ must be with one of the three fixed points. Since $a \neq \pi_{3}(b)$, the pair $\left\{b, \pi_{3}(b)\right\}$ can not appear with the point $\infty_{1}$. If $\left\{\infty_{0}, b, \pi_{3}(b)\right\} \in \mathcal{B}$, then we obtain a $(6,4)$-configuration $\left\{\infty_{0}, a, \pi_{3}(a)\right\},\left\{\infty_{0}, b, \pi_{3}(b)\right\},\left\{\infty_{1}, a, b\right\}$ and $\left\{\infty_{1}, \pi_{3}(a) \pi_{3}(b)\right\}$, a contradiction. Hence, we have $\left\{\infty_{2}, b, \pi_{3}(b)\right\} \in \mathcal{B}$, where $\infty_{2}$ is the remaining fixed point. But then we obtain a $(7,5)$-configuration $\left\{\infty_{0}, a, \pi_{3}(a)\right\},\left\{\infty_{1}, a, b\right\},\left\{\infty_{1}, \pi_{3}(a) \pi_{3}(b)\right\}$, $\left\{\infty_{2}, b, \pi_{3}(b)\right\}$ and $\left\{\infty_{0}, \infty_{1}, \infty_{2}\right\}$. This final contradiction completes the proof.

The following is an immediate corollary of the theorem above.

Corollary 3.2 For every $r \geq 5$, there exists no reverse $r$-sparse STS.

Since a 1-rotational STS is also reverse, we have:

Corollary 3.3 For every $r \geq 5$, there exists no 1-rotational $r$-sparse STS.

It is well known that the points and lines of $A G(n, 3)$ form the elements and triples of a 1-rotational, and thus reverse, 4-sparse $\operatorname{STS}\left(3^{n}\right)$. In this sense, the bounds of Theorem 3.1, Corollary 3.2 and 3.3 are best possible.

Corollary 3.3 limits the sparseness of a 1-rotational STS over any finite group even if it is nonabelian. The same bound for a rotational group action fixing three points inducing the other trivial subsystem follows from the same argument. However, if groups are restricted to be abelian, we can easily obtain a much stronger theorem. In fact, sparseness is limited to the lowest level.

Theorem 3.4 If the full automorphism group of an STS $S$ contains an abelian subgroup, which fixes more than one point and acts transitively on the other points, then $S$ is not 4-sparse.

Proof Let $S=(V, \mathcal{B})$ be an STS and $G$ be an abelian subgroup of the full automorphism group of $S$. Assume that $G$ fixes each point of $W \subset V$ and acts transitively on $V \backslash W$, where $|W| \geq 2$. Considering the neighbors of a point $a \in V \backslash W$ and two distinct fixed points $\infty_{0}, \infty_{1} \in W$, we obtain a pair of blocks $B_{0}=\left\{a, \infty_{0}, a^{i}\right\}$ and $B_{1}=\left\{a, \infty_{1}, a^{j}\right\}$. By developing $B_{1}$ and $B_{2}$, we obtain four blocks $B_{0}, B_{1}, B_{0}{ }^{j}$ and $B_{1}{ }^{i}$. Since $a=a^{i j} \Leftrightarrow a^{i}=a^{j} \Leftrightarrow \infty_{0}=\infty_{1}$, the family of the four blocks forms a $(6,4)$-configuration.

In the remainder of this paper we mention some corollaries on sparseness and automorphisms, similar to those we have just discussed.

An STS is said to be bicyclic if it admits a permutation on points, consisting of a pair of cycles of length $k$ and $v-k$, as an automorphism. Calahan and Gardner [5] 
proved that there exists a bicyclic $\operatorname{STS}(v)$ for $k>1$ if and only if $v \equiv 1,3(\bmod 6)$, $k \mid v$, and either $k \equiv 1(\bmod 6)$ and $3 k \mid v$, or $k \equiv 3(\bmod 6)$ and $k \neq 9$.

Corollary 3.5 Let $S$ be a bicyclic $r$-sparse STS and $l$ be the length of the smaller cycle of its bicyclic automorphism. Then

$$
r \leq \begin{cases}4 & \text { when } l=1,3 \\ 9 & \text { when } l \equiv 3 \bmod 6 \\ 12 & \text { when } l \equiv 1 \bmod 6\end{cases}
$$

Proof Let $\pi$ be a bicyclic automorphism of $S$ with the smaller cycle of length $l$. When $l=1, \pi$ consists of a fixed point and a cycle of length $v-1$. Then $S$ can be considered 1-rotational over $\boldsymbol{Z}_{v-1}$. When $l=3, \pi^{3}$ is an automorphism of $S$, consisting of three cycles of length $(v-3) / 3$ and three fixed points. Since $v$ is admissible and $3 \mid(v-3)$, we have $(v-3) / 3$ even. Hence, $S$ admits an involutive automorphism $\pi^{(v-3) / 2}$ fixing exactly three points. Hence, the bound for $l=1,3$ follows from Corollary 3.3 and Theorem 3.1.

Next we assume that $l \neq 1,3$. Let $W$ be the set of points in the point orbit of size $l$ under $\pi$. Consider a block $\{a, b, c\}$, where $a, b \in W$. Since $\pi^{l}$ fixes each point of $W$, we have $\{a, b, c\}=\pi^{l}(\{a, b, c\})$. Hence, $c=\pi^{l}(c)$ and we have $c \in W$. Hence, the points of $W$ induce a cyclic sub-STS of order $l$. The bounds for $l \equiv 1(\bmod 6)$ and $l \equiv 3(\bmod 6)$ follow from Corollary 2.4 .

An STS is said to be 1-transrotational if it admits an automorphism consisting one fixed point, a transposition and a cycle of length $v-3$. Gardner [17] showed that a 1-transrotational $\operatorname{STS}(v)$ exists if and only if $v \equiv 1,7,9,15(\bmod 24)$. Let $\pi$ be a 1-transrotational automorphism of an STS. Every 1-transrotational STS admits the involutive automorphism $\pi^{(v-3) / 2}$ fixing exactly three points, and hence it is at most 4-sparse by Theorem 3.1. In fact, a 1-transrotational STS contains many other $(7,5)$-configurations than those implied by Theorem 3.1 .

Corollary 3.6 For every $r \geq 5$, there exists no 1-transrotational $r$-sparse STS.

Proof Let $(V, \mathcal{B})$ be a 1-transrotational $\operatorname{STS}(v)$ and $\pi$ be a 1-transrotational automorphism fixing a point $\infty$. Since the unique STS $(7)$ contains a $(6,4)$-configuration, we assume that $v>7$. Then $\mathcal{B}$ contains at least one block of which each point lies in the point orbit of size $v-3$ under $\pi$. Write the block as $B_{1}=\left\{a, \pi^{i}(a), \pi^{j}(a)\right\}$. Take the block containing the pair $\{\infty, a\}$ and write it as $B_{2}=\left\{\infty, a, \pi^{k}(a)\right\}$. Then we obtain five blocks $B_{1}, \pi^{k}\left(B_{1}\right), B_{2}, \pi^{i}\left(B_{2}\right)$ and $\pi^{j}\left(B_{1}\right)$. Considering the neighbors of $\infty$, we have $\left|B_{1} \cup \pi^{k}\left(B_{1}\right) \cup B_{2} \cup \pi^{i}\left(B_{2}\right) \cup \pi^{j}\left(B_{1}\right)\right|=7$; otherwise, one of the three blocks $B_{2}, \pi^{i}\left(B_{2}\right)$ and $\pi^{j}\left(B_{1}\right)$ is reduced to a pair of points. Hence, we obtain a $(7,5)$-configuration.

Acknowledgements The author would like to thank Professor Mike Grannell, Professor Masakazu Jimbo, and Mr. Anthony D. Forbes for helpful discussions. He also thanks the anonymous referee for his careful reading and valuable comments. 


\section{References}

1. Beth, T., Jungnickel, D., \& Lenz, H. (1999). Design theory. Cambridge: Cambridge University Press.

2. Brouwer, A. E. (1977). Steiner triple systems without forbidden subconfigurations. Mathematisch Centrum Amsterdam, ZW 104/77.

3. Brown, W. G., Erdős, P., \& Sós, V. T. (1973). Some extremal problems on $r$-graphs. In F. Harary (Ed.), New directions in the theory of graphs, Proc. Third Ann Arbor Conf. Univ. Michigan, Ann Arbor, MI, October 1971 (pp. 53-63). New York: Academic Press.

4. Buratti, M. (2001). 1-rotational Steiner triple systems over arbitrary groups. J. Comb. Des., 9, 215226.

5. Calahan-Zijlstra, R., \& Gardner, R. B. (1994). Bicyclic Steiner triple systems. Discret. Math., 128, 35-44.

6. Chee, Y. M., Colbourn, C. J., \& Ling, A. C. H. (2000). Asymptotically optimal erasure-resilient codes for large disk arrays. Discret. Appl. Math., 102, 3-36.

7. Clapham, P. C. (1976). Steiner triple systems with block-transitive automorphism groups. Discret. Math., 14, 121-131.

8. Colbourn, C. J., \& Rosa, A. (1999). Triple systems. New York: Oxford University Press.

9. Colbourn, C. J., Mendelsohn, E., Rosa, A., \& Širáň, J. (1994). Anti-mitre Steiner triple systems. Graphs Comb., 10, 215-224.

10. Delandtsheer, A., Doyen, J., Siemons, J., \& Tamburini, C. (1986). Doubly homogeneous 2-( $v, k, 1)$ designs. J. Comb. Theory Ser. A, 43, 140-145.

11. Doyen, J. (1972). A note on Steiner triple systems. Discret. Math., 1, 315-319.

12. Erdős, P. (1976). Problems and results in combinatorial analysis. In Atti dei Convegni Lincei (Vol. 17, pp. 3-17). Colloquio Internazionale sulle Teorie Combinatorie, Tomo II, Rome, 1973. Rome: Accad. Naz. Lincei.

13. Forbes, A. D., Grannell, M. J., \& Griggs, T. S. (2007). On 6-sparse Steiner triple systems. J. Comb. Theory Ser. A, 114, 235-252.

14. Fujiwara, Y. (2005). Constructions for anti-mitre Steiner triple systems. J. Comb. Des., 13, 286-291.

15. Fujiwara, Y. (2006). Infinite classes of anti-mitre and 5-sparse Steiner triple systems. J. Comb. Des., $14,237-250$.

16. Fujiwara, Y. Cyclic 4- and 5-sparse Steiner triple systems (submitted).

17. Gardner, R. B. (1994). Steiner triple systems with transrotational automorphisms. Discret. Math., 131, 99-104.

18. Grannell, M. J., Griggs, T. S., \& Murphy, J. P. (1999). Some new perfect Steiner triple systems. J. Comb. Des., 7, 327-330.

19. Grannell, M. J., Griggs, T. S., \& Whitehead, C. A. (2000). The resolution of the anti-Pasch conjecture. J. Comb. Des., 8, 300-309.

20. Hall, M. Jr. (1985). Steiner triple systems with a doubly-transitive automorphism group. J. Comb. Theory Ser. A, 38, 192-202.

21. Hartman, A., \& Hoffman, D. G. (1987). Steiner triple systems with an involution. Eur. J. Comb., 8, 371-378.

22. Johnson, S. J., \& Weller, S. R. (2003). Resolvable 2-designs for regular low-density parity-check codes. IEEE Trans. Commun., 51, 1413-1419.

23. Kantor, W. M. (1985). Homogeneous designs and geometric lattices. J. Comb. Theory Ser. A, 38, 66-74.

24. Key, J. D., \& Shult, E. E. (1984). Steiner triple systems with doubly-transitive automorphism groups: a corollary to the classification theorem for finite simple groups. J. Comb. Theory Ser. A, 36, 105-110.

25. Kirkman, T. P. (1847). On a problem in combinations. Camb. Dublin Math. J., 2, 191-204.

26. Lefmann, H., Phelps, K. T., \& Rödl, V. (1993). Extremal problems for triple systems. J. Comb. Des., $1,379-394$.

27. Ling, A. C. H. (1997). A direct product construction for 5-sparse triple systems. J. Comb. Des., 5, 443-447.

28. Ling, A. C. H., Colbourn, C. J., Grannell, M. J., \& Griggs, T. S. (2000). Construction techniques for anti-Pasch Steiner triple systems. J. Lond. Math. Soc. (2), 61, 641-657.

29. Peltesohn, R. (1939). Eine Lösung der beiden Heffterschen Differenzenprobleme. Compos. Math., 6, 251-257.

30. Robinson, R. M. (1975). The structure of certain triple systems. Math. Comput., 29, 223-241.

31. Rosa, A. (1972). On reverse Steiner triple systems. Discret. Math., 2, 61-71.

32. Teirlinck, L. (1973). The existence of Steiner triple systems. Discret. Math., 6, 301-302. 
33. Vasic, B., \& Milenkovic, O. (2004). Combinatorial constructions of low-density parity-check codes for iterative decoding. IEEE Trans. Inf. Theory, 50, 1156-1176.

34. Vasic, B., Kurtas, E. M., \& Kuznetsov, A. V. (2002). Kirkman systems and their application in perpendicular magnetic recording. IEEE Trans. Mag., 38, 1705-1710.

35. Wolfe, A. (2005). 5-sparse Steiner triple systems of order $n$ exist for almost all admissible $n$. Electron. J. Comb., 12, Research Paper 68, 42 pp. (electronic).

36. Wolfe, A. (2006). The resolution of the anti-mitre Steiner triple system conjecture. J. Comb. Des., 14, $229-236$ 